\title{
Feasibility of a yoga, aerobic and stretching-toning exercise program for adult cancer survivors: the STAYFit trial
}

\author{
Neha P. Gothe $e^{1,2}$. Emily Erlenbach ${ }^{1}$
}

Received: 25 May 2021 / Accepted: 17 August 2021 / Published online: 28 August 2021

(C) The Author(s), under exclusive licence to Springer Science+Business Media, LLC, part of Springer Nature 2021

\begin{abstract}
Background The use of yoga as a mind-body practice has become increasingly popular among clinical populations and older adults who use this practice to manage age and chronic disease-related symptoms. Although yoga continues to gain popularity among practitioners and researchers, pilot studies that examine its feasibility and acceptability, especially among cancer survivors, are limited. Feasibility studies play a critical role in determining whether the target population is likely to engage with larger scale efficacy and effectiveness trials. In this paper we present feasibility and acceptability data from a 12-week randomized controlled trial (RCT) conducted with adult cancer survivors.

Methods Participants $n=78$ (Mean age: 55 years) were randomized to one of three groups: a Hatha yoga, aerobic exercise, or stretching-toning control group with group exercise classes held for $150 \mathrm{~min} /$ week for 12 weeks. Herein we report feasibility and acceptability, including enrollment rates, attendance, attrition and adverse events, and participant feedback and satisfaction data.

Results Of the 233 adults screened, 109 were eligible and 78 randomized to one of the three intervention arms. Session attendance was high for all groups (75.5-89.5\%) and 17 participants dropped out during the 12-week intervention. Program satisfaction was high (4.8 or higher out of 5) and no adverse events were reported. One cohort $(n=15)$ of the intervention transitioned to remote intervention delivery due to the COVID-19 pandemic. Feasibility data from these participants suggested that synchronized group exercise classes via Zoom with a live instructor were acceptable and enjoyable. Participant feedback regarding most and least helpful aspects of the program as well as suggestions for future yoga interventions are summarized.

Conclusions Overall, the yoga intervention was highly feasible and acceptable. The feasibility parameters from this trial can aid researchers in estimating recruitment rates for desired sample sizes to successfully randomize and retain cancer survivors in short- and long-term yoga-based efficacy and effectiveness trials. The findings also provide evidence to clinicians who can recommend up to $150 \mathrm{~min}$ of a combination of exercises-aerobic, yoga, or stretching-toning to their cancer patients in order to improve health and wellbeing during cancer survivorship.
\end{abstract}

Keywords Yoga intervention · Randomized controlled trial · Cancer survivorship · COVID-19

Neha P. Gothe

npg@illinois.edu

1 Department of Kinesiology and Community Health, University of Illinois At Urbana Champaign, Champaign, IL, USA

2 Beckman Institute for Advanced Science and Technology, Cancer Center At Illinois, Center for Social and Behavioral Sciences, University of Illinois At Urbana Champaign, Champaign, IL, USA

\section{Introduction}

Strong evidence supports the beneficial aspects of physical activity during and post cancer treatment $[1,2]$. The American Cancer Society (ACS) recommends that cancer survivors partake in regular physical activity, avoid inactivity, and return to normal daily activities as soon as possible after diagnosis [3]. Their recommendation aligns with the Physical Activity Guidelines for Americans which suggest adults engage in $150 \mathrm{~min}$ of moderate to vigorous intensity aerobic exercise in addition to two or more days of strength training [4]. 
More than 165 million people in the USA are cancer survivors. Early detection, improved health care, and advanced treatments have contributed to improved survival rates, with the 5-year relative survival rate at $67.7 \%$ for cancer patients [5]. Regular exercise has shown to improve a variety of health and wellbeing indicators among cancer survivors. These include improved cardiovascular fitness, muscle strength, body composition, fatigue, anxiety, depression, self-esteem, happiness, and several quality-of-life indicators [6]. Despite the abundance of research supporting exercise benefits during and after cancer, $34 \%$ of US adult cancer survivors reported no leisure time physical activity and only $15.7 \%$ of survivors actually met the federal guidelines discussed above [7]. In addition to common barriers to physical activity such as being busy, lack of motivation or willpower, and bad weather [8], cancer survivors face unique challenges caused by the diagnosis and treatment, such as increased depression, pain, or treatment-related fatigue $[9,10]$ that prevent them from engaging in exercise behavior. There is a need to test alternative non-pharmacological interventions to boost physical activity that would have greater appeal for this population given the unique barriers and challenges.

Yoga is a gentle and modifiable form of exercise that is increasingly popular among middle aged and older adults. While the intensity of yoga can vary depending on the style practiced and components of the practice (i.e., active poses vs. breathing and meditation), it is most commonly performed at a light-intensity level [11]. The recent National Health Interview Survey identified yoga as the most popular form of mind-body practice, used by $14.3 \%$ (32.5 million) of US adults [12]. Yoga and similar non-pharmacological alternative therapies are being increasingly used by adults for aging-related chronic conditions such as back pain, arthritis, anxiety, depression, and cancer [13-16]. A number of yogabased interventions have been conducted in the cancer survivorship literature and have shown evidence for improving mental, physical, social outcomes, as well as overall healthrelated quality of life $[17,18]$.

Recent feasibility trials for cancer survivors have focused on enhancing the physical activity experience through the use of motivational interviewing [19] and personalized coaching in the form of exercise counseling [20]. Other physical activity-based feasibility studies have focused on testing the use and uptake of technology such as the use of smartphone applications and social media [21-23], Fitbit [24], and interactive portals [25] to promote physical activity. Some have often focused on a specific cancer survivor group [19, 25-27]. While these studies have mostly reported improvements in cancer-related symptoms and quality of life, it still remains unknown if the recommended dosage of exercise is actually tolerable among this population.

In this manuscript, we summarize the feasibility parameters of designing and implementing a 12-week exercise and yoga intervention for adult cancer survivors. We report data from the STAYFit Trial conducted from August 2018 to May 2020 and feasibility parameters including recruitment, eligibility, and enrollment rates; adherence and attrition rate; and adverse events. We also collected self-reported data on participant's tolerability of the ACS/CDC recommendation of $150 \mathrm{~min}$ of exercise per week across the three different exercise styles and intensities $[4,29]$. Three cohorts were recruited to meet the target sample size and one cohort of the sample transitioned from in-person to fully remote exercise intervention for 7 of the 12 weeks of the intervention during the COVID-19 lockdown in March 2020. We also report the feasibility and acceptability of delivering an exercise intervention via synchronous technology-based (Zoom) group exercise interventions for the subset of our sample.

\section{Methods}

\section{Procedures and intervention}

Study design, participant eligibility criteria, and recruitment information have been previously published elsewhere [30]. In brief, the primary aim of STAYFit was to test the efficacy of a 12-week yoga program to improve cognitive function among cancer survivors compared with aerobic walking and a stretching-toning active control group. Low-active cancer survivors who participated in $\leq 2$ days per week of structured physical activity and were between the ages of 30 and 70 years were eligible. All cancer survivors (except brain) who had completed all cancer-related surgeries, radiation, or chemotherapy sessions at least 1 month prior to study enrollment were invited to participate. No eligibility restrictions were placed on the stage of individuals' cancer diagnosis. All enrolled participants were randomized into one of the three study arms: yoga, aerobic walking, or stretching-toning.

An in-depth description of the three intervention arms can be found in the published protocol paper [30]. All exercise groups met for 150 min per week. The yoga group practiced Hatha yoga, which focused on physical poses, breathing, and meditation and were led by a certified yoga instructor. The stretching-toning control arm participated in exercises targeting all major muscle groups and worked on balance, toning, and flexibility, with a greater focus on muscle strengthening compared with the yoga group. The aerobic group engaged in treadmill walking which increased in intensity and duration over the 12-week period and was individualized to each participant's aerobic fitness level.

The study was approved by the University of Illinois at Urbana Champaign's Institutional Review Board (IRB\# 18,922). All participants signed a written informed consent before completing any study assessments. Exercise sessions 
for the three exercise arms were held on the university campus (except for the latter half of the third cohort, which exercised in their homes due to COVID) in a group-exercise format. All in-person sessions were conducted in exercise studios equipped with necessary exercise equipment.

\section{Measures}

\section{Advertising and recruitment}

Due to the fact that this was a university-funded study with a modest budget, advertising efforts were limited to free or low-cost avenues. Methods of recruitment included emails through University of Illinois LISTSERV, contacting previous participants in the lab database who expressed interest in future studies, newspaper and radio ads, flyers and brochures posted around the community, social media postings, and booths at community events. To examine the efficacy of our recruitment efforts to attract potential participants, a recruitment rate was calculated. This metric was defined as the number of individuals who contacted the lab expressing interest in the study compared with the estimated total number of eligible individuals exposed to recruitment efforts, i.e., the number of individuals who were sent recruitment information. An eligibility rate was also calculated, which was the number of eligible individuals (based on screening criteria) divided by the total number of individuals who contacted the lab. Inversely, we also report a breakdown of ineligible participants and reasons for ineligibility. Additionally, we reported enrollment rate, which was the number of individuals randomized into the program compared with the total number who initially contacted the lab.

\section{Intervention data}

At the end of each exercise session over the entire study period, participants were asked to rank their enjoyment ("how much did you enjoy your activity session") and rating of perceived exertion (RPE, "how hard did you feel like you were working"). Enjoyment ratings were based off a 1-5 Likert scale, with 1 reflecting "did not enjoy at all" and 5 reflecting "enjoyed every much." RPE was based off the Borg 6-20 scale [31], with 6 reflecting "very, very light" and 20 reflecting "very, very hard." Weekly averages for each of these measures were computed to capture participants' overall tolerance of their respective exercise group.

\section{Attendance, attrition, and safety}

Overall attendance rates were calculated for each exercise group, and an independent samples $t$ test was run to test for group differences. Attendance rates for individual participants were calculated by dividing the number of exercise sessions attended by the total number of sessions in the 12-week program. Overall attendance rates for each exercise group were then calculated by averaging all participants' attendance rates in each group. Attrition rates, defined as the number of individuals who dropped out of the study divided by the total number of enrolled participants, were also calculated for each group. Cited reasons for study drop out were also summarized. Participants were asked about their general health and wellbeing weekly at the exercise sessions by the study staff. Any adverse events which occurred during the study period have also been reported.

\section{Participant program feedback}

At the end of the 12-week program, participants were asked to complete a program evaluation. On the form they were asked to rank the following items using a 1-5 Likert scale (1 reflecting "very dissatisfied" to 5 reflecting "very satisfied"): exercise program overall; progression of the exercise program; modifications of exercises and stretches; quality of exercise instruction; and quality of exercise program. Participant responses in each group were averaged to calculate an average score (ranging from 1 to 5) for each Likert question. Participants were also asked openended questions regarding areas of their life that had been impacted by STAYFit, most and least beneficial aspects of the program, and most helpful aspects to promote regular attendance and participation across the 12-week period.

\section{Feasibility and acceptability of Zoom-based exercise instruction}

In March 2020 the COVID-19 pandemic prevented the continuation of in-person exercise sessions for the third cohort of the study participants. In the face of this unforeseen event, the research team shifted the exercise sessions to an online format. Participants used Zoom (Zoom Video Communications) to follow along with live, virtual exercise instruction at their scheduled session times. Following the conclusion of this 12-week hybrid protocol, participants were asked additional questions relating to their experience using Zoom and engaging in virtual exercise classes. Participants were asked if they had ever engaged in a virtual exercise session using an electronic device (i.e., computer/tablet/smartphone). They were also asked to report their format of delivery preference (online sessions/in-person sessions/both), intensity of the online exercise sessions compared with in-person (same/easier/ harder), and enjoyment of the online sessions compared with in-person (same/more/less). 


\section{Results}

\section{Study recruitment}

Study recruitment occurred from September 2018 to December 2019. Figure 1 represents the study CONSORT. Data obtained from a marketing agency (AccuData) provided us with a baseline estimation of 1432 eligible cancer survivors in the targeted geographic area of ChampaignUrbana. Additionally, 347 cancer survivors from previous lab-based studies were contacted, producing an estimated number of 1779 potentially eligible cancer survivors exposed to recruitment efforts. Overall, 233 interested participants ( $87.55 \%$ female) contacted our lab, producing a recruitment rate of $13.10 \%$ (233/1779). Interested participants most frequently cited university LISTSERV emails as the method for obtaining study information.
One hundred and eleven $(111 / 233=47.64 \%)$ participants were excluded, with "too active" being the most prominent excluding criterion ( $n=42$ excluded). An additional $44(44 / 233=18.88 \%)$ individuals were excluded due to being unable to contact $(n=29)$, no longer being interested $(n=13)$, and failure to complete all baseline assessments $(n=2)$. Overall, out of the initial pool of interested participants, $78(78 / 233=33.48 \%)$ were officially enrolled and randomized in the trial.

\section{Baseline characteristics of study participants}

Table 1 summarizes the demographic and cancer characteristics of the 78 participants (mean age $=55.60$, $\mathrm{SD}=9.72$ ) randomized in this trial. Among the $n=78$, $85.90 \%(n=67)$ were female and $91.02 \%(n=71)$ were Caucasian. In addition to Caucasian individuals (who make up $63.14 \%$ of the Champaign-Urbana population),
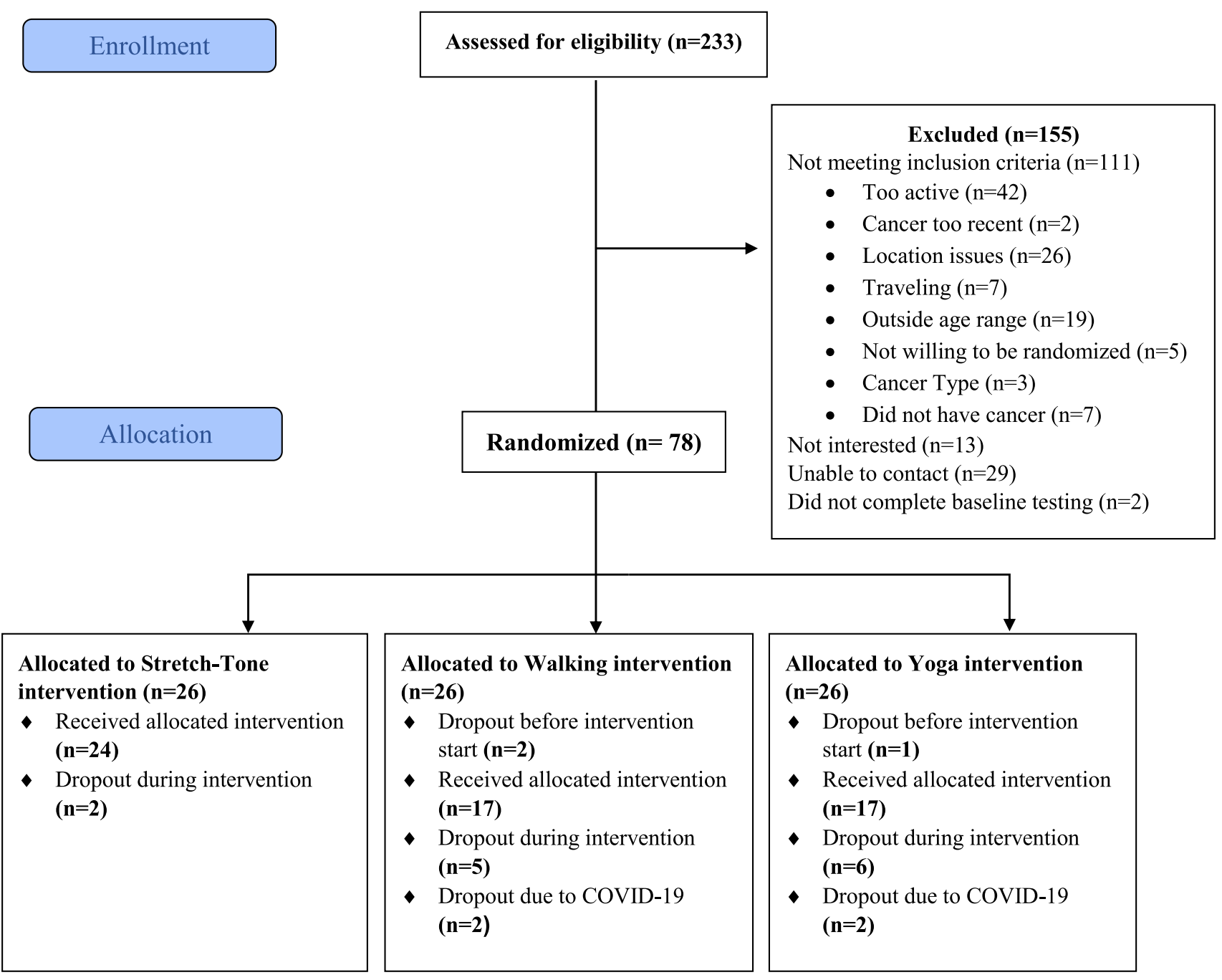

Fig. 1 Consort for the STAYFit trial 
Table 1 Demographic characteristics of the $N=78$ cancer survivors in the STAYFit trial

\begin{tabular}{|c|c|c|c|}
\hline & Walking $(n=26)$ & Yoga $(n=26)$ & Stretching $(n=26)$ \\
\hline Age (mean, SD) & $55.92(9.20)$ & $55.00(9.57)$ & $55.88(10.70)$ \\
\hline \multicolumn{4}{|l|}{$\operatorname{Sex}(n, \%)$} \\
\hline Females & $23(88.50)$ & $22(84.60)$ & $22(84.60)$ \\
\hline Males & $3(11.50)$ & $4(15.40)$ & $4(15.40)$ \\
\hline \multicolumn{4}{|l|}{$\operatorname{Race}(n, \%)$} \\
\hline African American & $2(7.70)$ & $1(3.80)$ & $2(7.70)$ \\
\hline More than one race & - & $1(3.80)$ & $1(3.80)$ \\
\hline Caucasian & $24(92.30)$ & $24(92.3)$ & $23.80(88.50)$ \\
\hline \multicolumn{4}{|l|}{ Education $(n, \%)$} \\
\hline$<$ College degree & $8(30.80)$ & $8(30.80)$ & $9(34.60)$ \\
\hline$\geq$ College degree & $18(69.20)$ & $18(69.20)$ & $17(65.40)$ \\
\hline \multicolumn{4}{|l|}{ Cancer type $(n, \%)$} \\
\hline Breast & $15^{*}(55.55)$ & $18(69.20)$ & $14(53.80)$ \\
\hline Cervical & - & $1(3.80)$ & $1(3.80)$ \\
\hline Colorectal & $1(3.70)$ & - & - \\
\hline Endometrial/uterine & - & $1(3.80)$ & - \\
\hline Leukemia & - & - & $2(7.70)$ \\
\hline Liver/bile duct & $1(3.70)$ & - & - \\
\hline Lung & $2(7.41)$ & - & - \\
\hline Lymphoma & $2(7.41)$ & - & $1(3.80)$ \\
\hline Multiple myeloma & - & $1(3.80)$ & - \\
\hline Ovarian & $3 *(11.11)$ & - & - \\
\hline Prostate & $1(3.70)$ & $2(7.70)$ & - \\
\hline Sarcoma & - & - & $1(3.80)$ \\
\hline Skin & $1(3.70)$ & $2(7.70)$ & $1(3.80)$ \\
\hline Thyroid & $1(3.70)$ & $1(3.80)$ & - \\
\hline \multicolumn{4}{|l|}{ Stage of cancer diagnosis $(n, \%)$} \\
\hline 0 & $1(3.38)$ & $1(3.85)$ & - \\
\hline 1 & $8(30.77)$ & $11(42.31)$ & $8(30.77)$ \\
\hline 2 & $8(30.77)$ & $8(30.77)$ & $8(30.77)$ \\
\hline 3 & $4(15.38)$ & $2(7.69)$ & $2(7.69)$ \\
\hline 4 & $2(7.69)$ & $1(3.85)$ & $2(7.69)$ \\
\hline Don't know & $3(11.54)$ & $3(11.54)$ & $6(23.08)$ \\
\hline $\begin{array}{l}\text { Years since diagnosis (mean, } \\
\text { SD) }\end{array}$ & $7.72(7.40)$ & $8.29(8.64)$ & $8.94(6.92)$ \\
\hline \multicolumn{4}{|l|}{ Cancer treatments $^{+}(n, \%)$} \\
\hline Surgery & $23(88.46)$ & $26(100.00)$ & $24(92.31)$ \\
\hline Radiation & $18(69.23)$ & $16(61.54)$ & $13(50.00)$ \\
\hline Chemotherapy & $13(50.00)$ & $12(46.15)$ & $13(50.00)$ \\
\hline
\end{tabular}

* One participant had both breast and ovarian diagnoses; percentages are calculated out of $n=26$ cases

+ Majority of participants reported undergoing more than one cancer treatment we recruited $n=5$ African Americans and $n=2$ participants reporting more than one race. Breast cancer was the most common cancer diagnoses among participants $(n=47)$, followed by skin $(n=4)$ and prostate $(n=3)$. The average time since diagnosis was $8.32( \pm 7.61)$ years. The average age was $55.92( \pm 9.2)$ for yoga participants, $55.00( \pm 9.57)$ for aerobic walking participants, and 55.88 $( \pm 10.69)$ for stretch-tone participants.

\section{Average weekly ratings of perceived exertion and enjoyment during exercise sessions}

Average weekly rating for perceived exertion for each group is displayed in Fig. 2. As expected, average perceived exertion ratings steadily increased among the aerobic walking group as the intensity and duration of the sessions increased. However, weekly average rating of perceived exertion among 
Fig. 2 Average weekly Rating of Perceived Exertion (RPE) for STAYFit participants across the 12-week study period

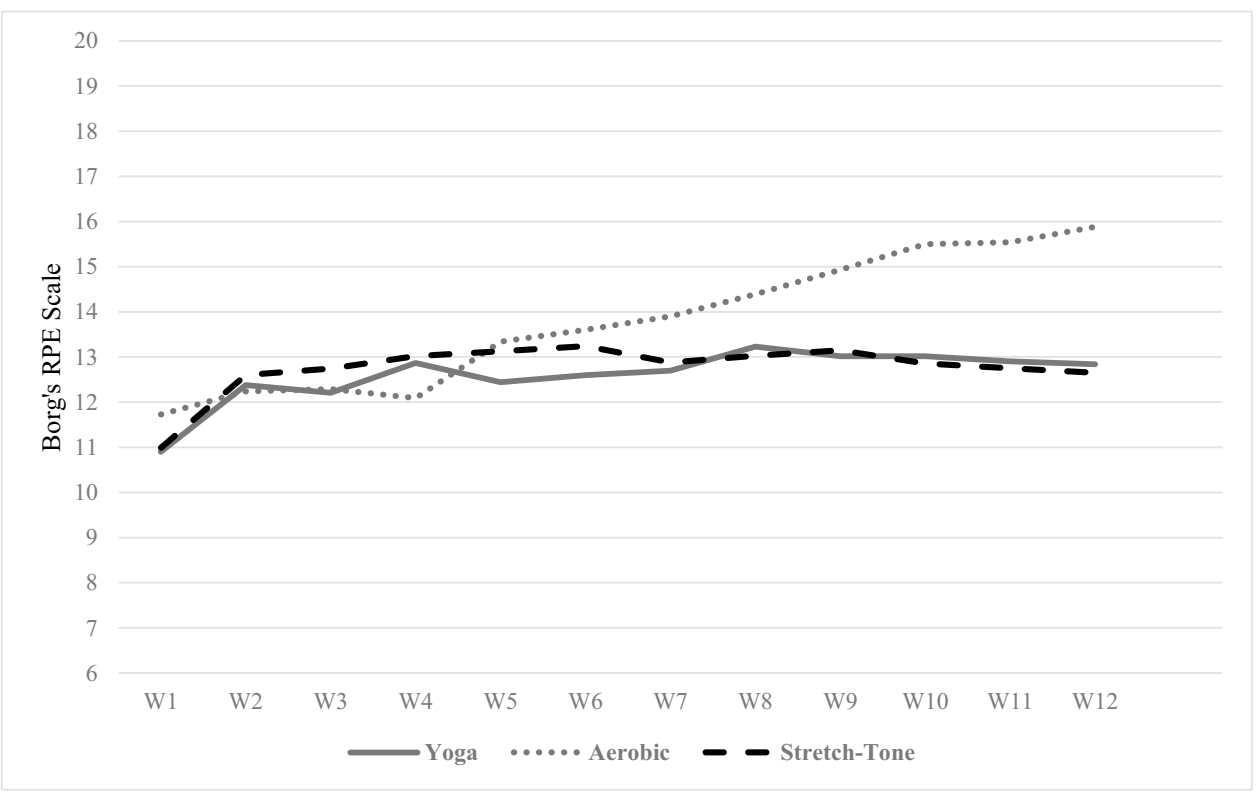

the yoga and stretch-tone arms remained constant and was similar across the entire study period. Overall, average enjoyment ratings were high and ranged from 3.63 (aerobic walking, week 10) to 4.56 (yoga, week 6).

\section{Attendance, attrition rates, and safety}

Both the aerobic walking and stretching-toning groups met three times per week for 1-h sessions for 12 weeks, culminating in 36 sessions. Average attendance was $89.53 \%$ for walking and $76.58 \%$ for the stretch-tone group. The yoga group met twice a week for 1.5-h sessions for 12 weeks (total of 24 sessions) and had an average attendance rate of $77.77 \%$.

Out of the 78 participants initially enrolled in the study, three dropped out after randomization but before starting the intervention. All three of these participants cited a change in their personal schedule preventing them from attending the scheduled group exercise sessions. Across the program, $n=13$ participants dropped out during the study intervention ( $n=2$ stretch-tone; $n=5$ aerobic; $n=6$ yoga participants). The most prevalent cited reason for discontinuation was health conditions/injuries unrelated to the study $(n=5)$, followed by personal reasons unrelated to the study $(n=3)$, changes in work schedule $(n=3)$, location issues $(n=1)$, and increased caregiving demands $(n=1)$. During the third study cohort, the transition from in-person to online instruction due to COVID-19 caused an additional four participants to drop out due to increased work demands as a frontline worker $(n=1)$, increased caregiving duties $(n=1)$, and lack of sufficient exercise space at home $(n=2)$. More importantly, no adverse events occurred in any of the groups over the entire study period.

\section{Participant program evaluation}

The STAYFit exercise program was highly rated by all participants, who on average ranked their "overall experience" as $4.66( \pm 0.51)$ out of 5 . Individual group ratings were 4.82 $( \pm 0.39)$ for yoga, $4.47( \pm 0.57)$ for aerobic walking, and $4.64( \pm 0.56)$ for stretch-tone participants which were not significantly different from each other ( $p$ values $>0.05$ ). Participants were also asked to rate the progression of their respective exercise program and average scores were 4.65 $( \pm 0.7)$ for yoga, $4.47( \pm 0.64)$ for aerobic, and $4.32( \pm 0.72)$ for stretch-tone participants. For "modifications of exercises and stretches," yoga participants gave an average rating of $4.59( \pm 0.62)$, aerobic participants an average rating of 4.40 $( \pm 0.74)$, and stretch-tone participants an average rating of $4.50( \pm 0.74)$. Regarding the "quality of exercise instruction," stretch-tone participants gave an average rating of 4.50 $( \pm 0.58)$, yoga participants gave an average rating of 4.82 $( \pm 0.39)$, and aerobic walking participants rated their instruction quality $4.53( \pm 0.64)$. Finally, "quality of the exercise program" was rated $4.53( \pm 0.58), 4.82( \pm 0.39)$, and 4.47 $( \pm 0.64)$ by stretch-tone, yoga, and aerobic participants, respectively.

Responses to the open-ended question about what areas of life STAYFit most impacted are listed in Table 2. Participants consistently reported having a regular exercise schedule/routine as the most impactful aspect of the program. Participants cited that the regular class session times provided "encouragement to stay with routine" and "reinforced exercise habits." Participants also reported gaining more knowledge about the importance of exercise and being exposed to new types of exercise (primarily yoga). Additionally, given that many of the study participants worked 
Table 2 Cancer survivors' self-reported health benefits following the 12-week exercise intervention

\begin{tabular}{llll}
\hline Physical & Mental & Social & Intrapersonal \\
\hline Overall health & Improved focus & Meeting new people & Motivation \\
Strength & Increased energy levels & Group exercise environment & Awareness of activity levels \\
Aerobic Fitness & Mental awareness & Something to do & Consistency \\
Pain reduction & Stress reductions & Being with other survivors & Accountability \\
Sleep quality & Improved mood & Establishing a work-life balance & Sense of giving back \\
Weight & Improved self-esteem & Good conversations & \\
Flexibility & Self-awareness & & \\
Balance & Enjoyment of program & & \\
Endurance & Mindfulness & & \\
Stamina & Confidence & & \\
Mobility & Body image & & \\
& Decreased anxiety & & \\
& Improved cognition & & \\
& Concentration & & \\
\hline
\end{tabular}

fulltime, achieving a better work-life balance with commitment to the exercise classes was also reported.

The most commonly reported beneficial aspects of STAYFit included the set schedule of exercise classes, increased accountability, and positive physical and mental changes. When asked to provide suggestions for future exercise programs, many participants expressed interest in extending the program by having exercises to follow along with at home or continuing longer than 12 weeks. Participants also recommended offering more options for class times to better accommodate work and life schedules. Lastly, participants expressed interest in sessions combining walking, stretchingtoning, and yoga in order to experience all three modalities.

\section{COVID-19 Zoom sessions}

Among cohort 3 participants whose study period was disrupted by COVID, $n=15$ were able to continue using the online format. Overall, 11 out of 15 participants reported that they had never used technology to participate in an online exercise class (such as using YouTube videos or other streaming services) and participating in the Zoom-based live exercise sessions was their first experience. Regarding preference, $60 \%(n=9)$ reported preferring the in-person sessions compared to $40 \%(n=6)$ who reported preferring both the online and in-person format. Despite a majority preferring the in-person format, $66.67 \%(n=10)$ said they enjoyed the online format the same as in-person. When asked about online session intensity, $66.67 \%(n=10)$ reported it felt the same, $20 \%(n=3)$ reported it was easier, and $13.33 \%(n=2)$ reported it was harder compared with in-person. Engagement during the online sessions also varied, with $46.67 \%$ $(n=7)$ reporting the same as in-person and $46.67 \%(n=7)$ reporting less than in-person; only $n=1$ individual reported feeling more engaged during online sessions.

\section{Discussion}

The primary aim of this paper was to report the feasibility of implementing a $150 \mathrm{~min} /$ week, 12-week exercise program for low-active cancer survivors. We simultaneously examined three unique modes of exercise-aerobic, stretching and toning, and yoga exercise to allow direct comparison in their tolerability and acceptance among participants. Both the individual session ratings and overall program feedback indicated that exercise doses of up to $150 \mathrm{~min} /$ week was well-tolerated for each of the exercise groups and participants reported experiencing positive health benefits. In spite of the strong evidence for the health benefits of physical activity for cancer survivors, small percentages of this population report being sufficiently active. This suggests the need for more feasibility studies to understand which elements of an exercise regimen are acceptable and well tolerated and therefore can be disseminated more widely in this clinical population.

Our work not only supports the feasibility of aerobic and stretching-toning exercise interventions but also adds to the literature by demonstrating that yoga is also a welltolerated form of exercise for this population. Independently, yoga, aerobic exercise, and stretching-toning have all been studied among cancer survivors [32]. Through the lenses of the FITT principle (frequency, intensity, type and time), we manipulated the "type" of exercise, to also test yoga, while holding the other components steady across groups. The Hatha yoga sessions were enjoyable and an acceptable form of exercise. Additionally, our participants were able to fully engage in all floor-based yoga movements and did not need any major modifications (i.e., chair-based yoga). Our participant experiences represent the national surveys that report the popularity and increasing use of yoga by adults, especially to manage chronic health conditions [33]. 
One of the main parameters of feasibility is recruitment and adherence rates in order to understand who is most likely to respond and take advantage of the study's opportunities and who will adhere to their participation in the study. It was surprising that, among the 233 interested people who reached out to the research staff, being "too active" was the most frequent criteria for ineligibility. "Responder bias" is a natural consequence of random sampling, and it's likely that people who responded to our recruitment efforts were already inclined to be physically active and/or were active and looking for ways to become more active. However, our enrollment rate of $33.47 \%$ was better than what has been previously reported. This could be due to the fact that we did not limit our participant pool to one specific type of cancer. Feasibility studies among cancer survivors discussed in the introduction of this manuscript with similar community-wide recruitment strategies have reported similar enrollment rates of 9.33-35.38\% [25, 26]. However, it is also fairly common for feasibility studies with specific cancer types to conduct targeted recruitment through clinics and oncology centers using referrals from oncologists, thus reporting higher enrollment rates [19, 25-27]. Our recruitment efforts without any collaboration with a clinic yielded a good sample size suggesting that this population is indeed interested in physical activity programs and 12 weeks of structured exercise up to $3 \mathrm{~h} /$ week was not perceived as a burden or challenge.

While online-exercise delivery was not part of the original study protocol, the COVID-19 pandemic in March 2020 forced us to adapt our program for a subset of the study participants. However, this gave us a unique opportunity to compare the feasibility of online vs in-person exercise sessions. Although technology and fitness-based apps are widely available $[34,35]$ and have been the foundation of previous physical activity-based studies [36,37], a majority of our sample had never used a virtual platform for exercise. Although the participants preferred exercising as a group inperson, the virtual sessions were equally enjoyable for the participants. This is significant as virtual programs are more likely to be disseminable and can reach a larger population of cancer survivors, thereby having a larger public health impact. On the other hand, we also had four participants who could not continue with their participation in the trial when we transitioned to virtual sessions due to being frontline workers, taking care of family members, and lack of sufficient exercise space in their household. These factors should also be taken into consideration for future studies that are designed to promote home-based physical activity interventions for cancer survivors as well as other clinical populations. However, it is worth noting that the individuals who were unable to continue participation due to COVIDinduced barriers may still be able to engage in home-based exercise sessions under more normal circumstances.

\section{Strengths and limitations}

Although we were able to reach out target sample size, a potentially limiting factor was our inability to directly recruit through local hospitals, clinics, and cancer centers. Previous studies have reported how essential it is to work with clinics and have participants' attending physicians encourage them to engage in such programs [38]. Researchers in non-clinical academic settings that are not affiliated with a hospital or clinic need to engage in productive partnerships to develop truly translational and disseminable programs. Our sample was heterogeneous in that we included participants with various cancer types and stages in the study. However, Caucasian, female breast cancer survivors still made up a large majority of our sample. Recruitment of male participants, especially in yoga trials can be a challenge [39], and national surveys tend to report greater participation of females in yoga practice than males [33,40]. Lastly, given that many of our participants were quite a few years removed from their initial cancer diagnosis and treatment, we were unable to specifically observe and compare the benefits of the STAYFit program on survivors who more recently completed their treatments.

\section{Conclusion}

The present study compared the feasibility and acceptability of a 150 min per week, 12-week exercise intervention of three exercise modes-aerobic, stretching-toning, and yoga among inactive cancer survivors. Our enrollment, eligibility and retention rates were comparable to past studies, and no adverse events were reported across the trial duration. Our results demonstrate that all three exercise arms of our STAYFit RCT were well-tolerated by cancer survivors. It should also be noted that a majority of the available evidence on the safety and efficacy of exercise during and after cancer treatment is derived from RCTs conducted with breast cancer survivors [41]. This is one of the first trials to simultaneously compare the acceptability of three exercise modes among a heterogeneous sample of inactive cancer survivors. Based on the current literature, moderate-intensity aerobic training at least $30 \mathrm{~min}, 3 \mathrm{x} / \mathrm{week}$, for at least $8-12$ weeks is considered to be an effective exercise prescription that most consistently addresses health-related outcomes experienced due to a cancer diagnosis and cancer treatment [41]. Our RCT supports the existing evidence for the feasibility of aerobic training but also provides preliminary support to further test the efficacy and effectiveness of the popular alternative of yoga practice for cancer survivorship. The recruitment and enrollment rates from our trial can aid researchers to power larger trials to examine the full scope and mechanisms behind the health benefits of yoga exercise. 
Acknowledgements The authors would like to thank all the participants who volunteered their time and energy for this study and the research staff who assisted with delivery of the exercise intervention and data management. The authors would also like to thank yoga instructor Linda Lehovec for her instruction and engagement with the trial and Sam Streeter for project coordination.

Authors' contributions NG: conceptualized the trial, secured funding, supervised execution of the trial; data management and analysis, and revised the manuscript. EE: assisted with execution of the trial, data collection and management, data analysis, and writing of the manuscript.

Funding This study was funded by the Office of the Vice Chancellor for Research, Campus Research Board Award RB\#18107, University of Illinois at Urbana Champaign.

\section{Declarations}

Ethical approval All protocols performed in this human subjects research study involving were in accordance with the ethical standards of the University of Illinois Institutional Review Board and with the 1964 Helsinki declaration and its later amendments or comparable ethical standards.

Informed consent All participants signed a written informed consent prior to any study measurement and procedures.

Consent for publication The study contains no individual person's data in any form.

\section{ClinicalTrials.gov Identifier NCT03650322}

Conflict of interest The authors declare that they have no conflict of interest.

\section{References}

1. Hilfiker R, Meichtry A, Eicher M, Balfe LN, Knols RH, Verra ML, et al. Exercise and other non-pharmaceutical interventions for cancer-related fatigue in patients during or after cancer treatment: a systematic review incorporating an indirect-comparisons metaanalysis. Br J Sports Med. BMJ Publishing Group Ltd and British Association of Sport and Exercise Medicine; 2018;52:651-8.

2. Courneya KS. Exercise in cancer survivors: an overview of research. Med Sci Sports Exerc United States. 2003;35:1846-52.

3. Physical Activity and the Cancer Patient [Internet]. Available from: https://www.cancer.org/treatment/survivorship-during-andafter-treatment/staying-active/physical-activity-and-the-cancerpatient.html. Accessed 2 Aug 2021.

4. Physical Activity Guidelines for Americans, 2nd edition. [Internet]. Washington, DC: U.S.; 2018. Available from: https://health. gov/sites/default/files/2019-09/Physical_Activity_Guidelines_ 2nd_edition.pdf. Accessed 2 Aug 2021.

5. National Cancer Institute. Cancer stat facts: cancer of any site. [Internet]. Surveillance, Epidemiol. End Results Progr. 2018 [cited 2021 Apr 15]. Available from: https://seer.cancer.gov/statf acts/html/all.html

6. Cramp F, Daniel J. Exercise for the management of cancerrelated fatigue in adults. Cochrane database Syst Rev. England; 2008;CD006145.
7. National Cancer Institute. Cancer Trends Progress Report: Cancer Survivors and Physical Activity [Internet]. 2020. [cited 2021 Apr 15]. Available from: https://progressreport.cancer.gov/after/physi cal_activity. Accessed 2 Aug 2021.

8. Ottenbacher AJ, Day RS, Taylor WC, Sharma SV, Sloane R, Snyder DC, et al. Exercise among breast and prostate cancer survivors-what are their barriers? J Cancer Surviv. 2011;5:413-9.

9. Oyekanmi G, Paxton RJ. Barriers to physical activity among African American breast cancer survivors. Psychooncology [Internet]. 2014/03/18. 2014;23:1314-7. Available from: https://pubmed. ncbi.nlm.nih.gov/24644092. Accessed 2 Aug 2021.

10. Eng L, Pringle D, Su J, Shen XW, Mahler M, Niu C, et al. Patterns, perceptions, and perceived barriers to physical activity in adult cancer survivors. Support Care Cancer. Supportive Care in Cancer; 2018;26:3755-63.

11. Larson-Meyer DE. A Systematic review of the energy cost and metabolic intensity of yoga. Med Sci Sports Exerc United States. 2016;48:1558-69.

12. Clarke T, Barnes P, Black L, Stussman B, Nahin R. Use of yoga, meditation, and chiropractors among U.S. adults aged 18 and over. [Internet]. Hyattsville, MD: National Center for Health Statistics; 2018. Available from: https://www.cdc.gov/nchs/data/databriefs/ db325-h.pdf. Accessed 2 Aug 2021.

13. Cherniack EP, Senzel RS, Pan CX. Correlates of use of alternative medicine by the elderly in an urban population. J Altern Complement Med United States. 2001;7:277-80.

14. Flaherty JH, Takahashi R, Teoh J, Kim JI, Habib S, Ito M, et al. Use of alternative therapies in older outpatients in the United States and Japan: prevalence, reporting patterns, and perceived effectiveness. J Gerontol A Biol Sci Med Sci. United States; 2001;56:M650-5.

15. Eisenberg DM, Davis RB, Ettner SL, Appel S, Wilkey S, Van Rompay M, et al. Trends in alternative medicine use in the United States, 1990-1997: results of a follow-up national survey. JAMA United States. 1998;280:1569-75.

16. Astin JA, Pelletier KR, Marie A, Haskell WL. Complementary and alternative medicine use among elderly persons: one-year analysis of a Blue Shield Medicare supplement. J Gerontol A Biol Sci Med Sci. United States; 2000;55:M4-9.

17. Buffart LM, van Uffelen JGZ, Riphagen II, Brug J, van Mechelen W, Brown WJ, et al. Physical and psychosocial benefits of yoga in cancer patients and survivors, a systematic review and metaanalysis of randomized controlled trials. BMC Cancer Springer. 2012;12:1-21

18. Culos-Reed SN, Mackenzie MJ, Sohl SJ, Jesse MT, Zahavich ANR, Danhauer SC. Yoga \& cancer interventions: a review of the clinical significance of patient reported outcomes for cancer survivors. Evidence-Based Complement Altern Med. Hindawi; 2012;2012.

19. Lion A, Backes A, Duhem C, Ries F, Delagardelle C, Urhausen A, et al. Motivational interviewing to increase physical activity behavior in cancer patients: a pilot randomized controlled trials. Integr Cancer Ther [Internet]. SAGE Publications; 2020;19:1534735420914973-1534735420914973. Available from: https://pubmed.ncbi.nlm.nih.gov/32202163. Accessed 2 Aug 2021.

20. Trinh L, Kramer AF, Rowland K, Strom DA, Wong JN, McAuley E. A pilot feasibility randomized controlled trial adding behavioral counseling to supervised physical activity in prostate cancer survivors: behavior change in prostate cancer survivors trial (BOOST). J Behav Med United States. 2021;44:172-86.

21. Pope Z, Lee JE, Zeng N, Lee HY, Gao Z. Feasibility of smartphone application and social media intervention on breast cancer survivors' health outcomes. Transl Behav Med England. 2019;9:11-22. 
22. Ritvo P, Obadia M, Santa Mina D, Alibhai S, Sabiston C, Oh $\mathrm{P}$, et al. Smartphone-Enabled Health Coaching Intervention (iMOVE) to promote long-term maintenance of physical activity in breast cancer survivors: protocol for a feasibility pilot randomized controlled trial. JMIR Res Protoc. 2017;6:e165.

23. Gell NM, Grover KW, Humble M, Sexton M, Dittus K. Efficacy, feasibility, and acceptability of a novel technology-based intervention to support physical activity in cancer survivors. Support care cancer Off J Multinatl Assoc Support Care Cancer. Germany; 2017;25:1291-300.

24. Rossi A, Frechette L, Miller D, Miller E, Friel C, Van Arsdale A, et al. Acceptability and feasibility of a Fitbit physical activity monitor for endometrial cancer survivors. Gynecol Oncol United States. 2018;149:470-5.

25. Kuijpers W, Groen WG, Oldenburg HS, Wouters MW, Aaronson NK, van Harten WH. eHealth for Breast Cancer Survivors: Use, Feasibility and Impact of an Interactive Portal. JMIR cancer. 2016;2:e3.

26. Peddle-McIntyre CJ, Baker MK, Lee YCG, Galvão DA, Cormie $\mathrm{P}$, Graham V, et al. The feasibility of a pragmatic distance-based intervention to increase physical activity in lung cancer survivors. Eur J Cancer Care (Engl). England; 2018;27.

27. Pullen T, Sharp P, Bottorff JL, Sabiston CM, Campbell KL, Ellard SL, et al. Acceptability and satisfaction of project MOVE: A pragmatic feasibility trial aimed at increasing physical activity in female breast cancer survivors. Psychooncology. 2018;27:1251-6.

28. De Jesus S, Fitzgeorge L, Unsworth K, Massel D, Suskin N, Prapavessis H, et al. Feasibility of an exercise intervention for fatigued breast cancer patients at a community-based cardiac rehabilitation program. Cancer Manag Res. 2017;9:29-39.

29. Rock CL, Thomson C, Gansler T, Gapstur SM, McCullough ML, Patel A V, et al. American Cancer Society guideline for diet and physical activity for cancer prevention. CA Cancer J Clin [Internet]. American Cancer Society; 2020;70:245-71. Available from: https://doi.org/10.3322/caac.21591

30. Gothe NP, Erlenbach ED, Streeter SL, Lehovec L. Effects of yoga, aerobic, and stretching and toning exercises on cognition in adult cancer survivors: protocol of the STAY Fit pilot randomized controlled trial. Trials. 2020;21:792.

31. Borg GA. Psychophysical bases of perceived exertion. Med Sci Sport Exerc [Internet]. 1982;14. Available from: https://doi.org/ 10.1249/00005768-198205000-00012

32. Duncan M, Moschopoulou E, Herrington E, Deane J, Roylance $\mathrm{R}$, Jones L, et al. Review of systematic reviews of non-pharmacological interventions to improve quality of life in cancer survivors. BMJ Open. 2017;7:e015860.

33. Ross A, Friedmann E, Bevans M, Thomas S. National survey of yoga practitioners: Mental and physical health benefits.
Complement Ther Med [Internet]. Churchill Livingstone; 2013 [cited 2019 Aug 13];21:313-23. Available from: https://www. sciencedirect.com/science/article/pii/S09652299130006 30. Accessed 2 Aug 2021.

34. Martín Payo R, Harris J, Armes J. Prescribing fitness apps for people with cancer: a preliminary assessment of content and quality of commercially available apps. J Cancer Surviv United States. 2019;13:397-405.

35. Roberts AL, Potts HW, Koutoukidis DA, Smith L, Fisher A. Breast, Prostate, and Colorectal Cancer Survivors' Experiences of Using Publicly Available Physical Activity Mobile Apps: Qualitative Study. JMIR mHealth uHealth. 2019;7:e10918.

36. Haberlin C, O'Dwyer T, Mockler D, Moran J, O'Donnell DM, Broderick J. The use of eHealth to promote physical activity in cancer survivors: a systematic review. Support care cancer Off J Multinatl Assoc Support Care Cancer. Germany; 2018;26:3323-36.

37. Laplante C, Peng W. A systematic review of e-health interventions for physical activity: an analysis of study design, intervention characteristics, and outcomes. Telemed $\mathbf{J}$ e-health Off $\mathbf{J}$ Am Telemed Assoc. United States; 2011;17:509-23.

38. Yates JIW. Clinicians in community practice are major contributors to clinical trials in cancer prevention, early detection, and treatment. CA. Cancer J. Clin. United States; 2003. p. 69-72.

39. Gothe NP, Kramer AF, McAuley E. The effects of an 8-week Hatha yoga intervention on executive function in older adults. Journals Gerontol Ser A Biol Sci Med Sci. Oxford University Press; 2014;69:1109-16.

40. Cartwright T, Mason H, Porter A, Pilkington K. Yoga practice in the UK: a cross-sectional survey of motivation, health benefits and behaviours. BMJ Open [Internet]. Br Med J Publ Group; 2020;10. Available from: https://bmjopen.bmj.com/content/10/1/ e031848. Accessed 2 Aug 2021.

41. Campbell KL, Winters-stone KM, Wiskemann J, May AM, Schwartz AL, Courneya KS, et al. Exercise Guidelines for Cancer Survivors: Consensus Statement from International Multidisciplinary Roundtable. Med Sci Sport Exerc [Internet]. 2019;51. Available from: https://journals.lww.com/acsm-msse/Fulltext/ 2019/11000/Exercise_Guidelines_for_Cancer_Survivors_.23. aspx. Accessed 2 Aug 2021.

Publisher's note Springer Nature remains neutral with regard to jurisdictional claims in published maps and institutional affiliations. 